\title{
Coupled-oscillator systems: efficient simulation with harmonic-balance based oscillator models.
}

\author{
Almudena Suárez, Franco Ramírez, Sergio Sancho \\ University of Cantabria \\ Santander, Spain
}

\begin{abstract}
A two-level simulation methodology for coupledoscillator systems is presented. The inner level corresponds to the harmonic-balance analysis of the elementary oscillator in freerunning regime, so as to obtain a one-port reduced-order model. The outer level corresponds to the analysis of the coupled system, deriving a formulation that explicitly relates the oscillationfrequency deviation and the amplitude and phase distributions to magnitudes characterizing the coupling network and oscillator elements. A stability analysis based on this formulation is also carried out obtaining a mathematical condition that determines the stable phase-shift interval. The formulation provides valuable insight into the system behavior and allows the derivation of realistic criteria for an optimum coupled-system performance. The two-level analysis has been extended to injection-locked systems, which enables an investigation of the impact of the number of oscillator elements and relative position of the injection signal on the locking bandwidth.
\end{abstract}

Keywords-Coupled-oscillator system, stability analysis, phase noise analysis, near carrier noise

\section{INTRODUCTION}

Coupled-oscillator systems can be used for beam steering of phased-array antennas and spatial-power combination [1-3]. As shown in [1-3], in a one-dimensional array, a constant phase-shift distribution can be obtained detuning the outermost oscillators only. The analysis of these systems often relies on simple oscillator models, of the Van der Pol type. In [4] a harmonic-balance (HB) analysis, using an auxiliary generator (AG) per oscillator element (to sustain the individual oscillations) has been presented. However, this circuit-level simulation becomes computationally unaffordable for high number of oscillator elements. The works [5-6] proposed the use of a reduced-order model of the free-running oscillator, obtained from a HB simulation, which extends the technique in [7], (previously applied to frequency dividers) to coupledoscillator systems. This enables a two-level simulation of these systems, such that the inner level corresponds to a HB analysis of the individual oscillator in free-running regime and the outer level corresponds to the analysis of the coupled system using the HB-oscillator models. By means of a first-order approximation of the coupled-system equations (such as the one applied in [1-3] to the Van-der-Pol models), it is possible to derive insightful expressions that explicitly relate the coupled-system oscillation frequency and the amplitude and phase distributions to magnitudes characterizing the coupling network and oscillator elements. A key aspect is that, as will be demonstrated, the steady-state response of a system with $n$ oscillator elements can be inferred from that of a system of only three elements. Taking the stability properties into account, mathematical criteria are derived for an optimum system performance, with minimum frequency deviation and unambiguous excursions of the tuning parameters. The methodology will be extended to coupled-oscillator systems with an injection-locking signal [8]. This will enable an understanding of the impact of the number of oscillator elements and relative positions of the injection signal on the locking bandwidth.

\section{TWO-LEVEL ANALYSIS}

The coupled-oscillator system is analyzed with a two-level procedure, described in the following.

\section{A. Inner level}

When isolated from the coupled system, the total admittance function of the oscillator circuit (current-to-voltage ratio) at any analysis node is equal to zero. When introduced into the coupled system, this admittance function will be different from zero since there will be current entering through the coupling networks (Fig. 1). However, provided this current has small amplitude (as in the most common case of weak coupling) it will only undergo a small variation. Therefore, it can be modeled with a first-order Taylor series about the freerunning solution. Thus, each oscillator element will be described with the partial derivatives of the admittance function $Y$ (current-to-voltage ratio) with respect to the amplitude $V$, frequency $\omega$ and tuning parameter $\eta$, respectively given by $\mathrm{Y}_{\mathrm{V}}, \mathrm{Y}_{\omega}, \mathrm{Y}_{\eta}$. These derivatives are calculated introducing a voltage AG in the isolated oscillator [7], at the same node where the coupling network should be connected at a later stage (Fig. 1). At the quiescent point, the AG frequency agrees with the oscillation frequency and its amplitude $\mathrm{A}_{\mathrm{AG} 0}$ agrees with the free-running node-voltage amplitude. The admittance-function derivatives are calculated through finitedifferences. For instance, the derivative $Y_{V}$ is calculated keeping the AG frequency and tuning voltage at their steadystate variables and performing a small sweep in the amplitude $\mathrm{A}_{\mathrm{AG}}$ about the free-running value $\mathrm{A}_{\mathrm{AG} 0} \equiv \mathrm{V}_{0}$ [7]. This technique has been applied to the individual oscillator of Fig. 1 at $5.22 \mathrm{GHz}$, which is based on the FET NE3210S01. It provides the derivatives: $\quad \mathrm{Y}_{\mathrm{V}}=0.044 \angle-5.75^{\circ} \Omega^{-1} / \mathrm{V}$, $\mathrm{Y}_{\omega}=2 \cdot 10^{-11} \angle 47^{\circ} \Omega^{-1} / \mathrm{rad}$ and $\mathrm{Y}_{\eta}=0.0068 \angle-140^{\circ} \Omega^{-1} / \mathrm{V}$. 


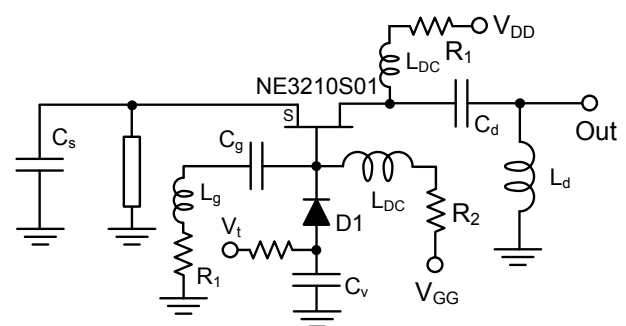

(a)

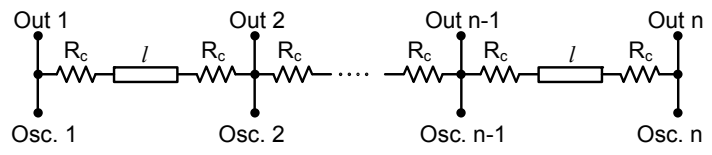

(b)

Fig. 1 Coupled-oscillator system with $\mathrm{n}$ oscillator elements. (a) Elementary oscillator at 5.22 GHz, based on the FET NE3210S01. (b) Coupling network.

\section{B. Outer level}

The oscillators are coupled by means of equal sub-networks [1-3], which generally consist of a transmission-line section bounded with series resistors (Fig. 1). Each sub-network is described by a symmetrical $2 \times 2$ admittance matrix $\mathrm{Y}_{\mathrm{c} 2}$ :

$$
Y_{c 2}=\left[\begin{array}{cc}
Y_{e} & Y_{n b} \\
Y_{n b} & Y_{e}
\end{array}\right]
$$

Applying Kirchoff's laws and assuming a constant interstage phase shift $\phi$, one obtains, for $\mathrm{n}$ oscillator elements, the following system in the increment $\Delta \omega$ with respect to the freerunning frequency, and the increments $\Delta \mathrm{V}_{\mathrm{i}}, \Delta \eta_{\mathrm{i}}(\mathrm{i}=1$ to $\mathrm{n}$ ) with respect to the free-running amplitude and tuning parameter:

$$
\begin{aligned}
& \mathrm{Y}_{\mathrm{V}} \Delta \mathrm{V}_{1}+\mathrm{Y}_{\omega} \Delta \omega+\mathrm{Y}_{\eta} \Delta \eta_{1}=-\mathrm{Y}_{\mathrm{e}}-\mathrm{Y}_{\mathrm{nb}} \mathrm{e}^{\mathrm{j} \phi} \equiv \mathrm{Y}_{1}^{\mathrm{c}}(\phi), \\
& \mathrm{Y}_{\mathrm{V}} \Delta \mathrm{V}_{2}+\mathrm{Y}_{\omega} \Delta \omega=-\mathrm{Y}_{\mathrm{nb}} \mathrm{e}^{-\mathrm{j} \phi}-2 \mathrm{Y}_{\mathrm{e}}-\mathrm{Y}_{\mathrm{nb}} \mathrm{e}^{\mathrm{j} \phi} \equiv \mathrm{Y}^{\mathrm{c}}(\phi) \\
& \vdots \\
& \mathrm{Y}_{\mathrm{V}} \Delta \mathrm{V}_{\mathrm{n}-1}+\mathrm{Y}_{\omega} \Delta \omega+\mathrm{Y}_{\eta} \Delta \eta_{\mathrm{n}-1}=-\mathrm{Y}_{\mathrm{nb}} \mathrm{e}^{-\mathrm{j} \phi}-2 \mathrm{Y}_{\mathrm{e}}-\mathrm{Y}_{\mathrm{nb}} \mathrm{e}^{\mathrm{j} \phi} \equiv \mathrm{Y}^{\mathrm{c}}(\phi) \\
& \mathrm{Y}_{\mathrm{V}} \Delta \mathrm{V}_{\mathrm{n}}+\mathrm{Y}_{\omega} \Delta \omega+\mathrm{Y}_{\eta} \Delta \eta_{\mathrm{n}}=-\mathrm{Y}_{\mathrm{nb}} \mathrm{e}^{-\mathrm{j} \phi}-\mathrm{Y}_{\mathrm{e}} \equiv \mathrm{Y}_{\mathrm{n}}^{\mathrm{c}}(\phi),
\end{aligned}
$$

To balance the system, an arbitrary tuning parameter $\Delta \eta_{2}$ is set to zero. Note that according to [1-3] the inner parameters will only undergo negligible variations, since the constant inter-stage phase shift is varied by detuning the two outermost oscillators only. The outer-level system (2) is nonlinear in the phase shift, but linear in the amplitude and tuning-voltage increments. Splitting system (2) into real and imaginary parts, this system can be written in a compact manner as $\mathrm{M}_{\mathrm{X}} \overline{\mathrm{X}}=\overline{\mathrm{Y}}_{\mathrm{c}}(\phi), \quad$ with $\quad \overline{\mathrm{X}}=\left[\Delta \omega_{\mathrm{s}}, \Delta \mathrm{V}_{1}, \cdots, \Delta \mathrm{V}_{\mathrm{N}}, \Delta \eta_{1}, \cdots, \Delta \eta_{\mathrm{N}}\right]^{\mathrm{t}}$. Solving for $\Delta \omega$ :

$$
\begin{aligned}
& \Delta \omega=\frac{\left(\mathrm{Y}_{\mathrm{V}} \times \mathrm{Y}_{\eta}\right)^{\mathrm{n}-1}\left(\mathrm{Y}_{\mathrm{V}} \times \mathrm{Y}^{\mathrm{c}}(\phi)\right)}{\left(\mathrm{Y}_{\mathrm{V}} \times \mathrm{Y}_{\eta}\right)^{\mathrm{n}-1}\left(\mathrm{Y}_{\mathrm{V}} \times \mathrm{Y}_{\omega}\right)}=\Delta \omega_{0}-\frac{2\left|\mathrm{Y}_{\mathrm{nb}}\right| \sin \alpha_{\mathrm{v}, \mathrm{nb}}}{\left|\mathrm{Y}_{\omega}\right| \sin \alpha_{\mathrm{v}, \omega}} \cos \phi \\
& \Delta \omega_{0}=-2\left(\left|\mathrm{Y}_{\mathrm{e}}\right| \sin \alpha_{\mathrm{v}, \mathrm{e}} /\left|\mathrm{Y}_{\omega}\right| \sin \alpha_{\mathrm{v}, \omega}\right)
\end{aligned}
$$

where $\times$ indicates the cross product of real and imaginary parts and $\alpha_{a, b}=$ phase $\left(Y_{b}\right)-$ phase $\left(Y_{a}\right)$. On the other hand, when solving (2) for $\Delta \eta_{1}$ one obtains:

$$
\begin{aligned}
& \Delta \eta_{1}=\frac{\left(Y_{V} \times Y_{\eta}\right)^{n-2}\left(Y_{V} \times Y_{\omega}\right)\left(Y_{V} \times\left(Y_{1}^{c}(\phi)-Y^{c}(\phi)\right)\right)}{\left(Y_{V} \times Y_{\eta}\right)^{n-1}\left(Y_{V} \times Y_{\omega}\right)}= \\
& =\frac{\left|Y_{e}\right| \sin \alpha_{v, e}}{\left|Y_{\eta}\right| \sin \alpha_{v, \eta}}-\frac{\left|Y_{n b}\right| \sin \left(-\phi+\alpha_{v, n b}\right)}{\left|Y_{\eta}\right| \sin \alpha_{v, \eta}}
\end{aligned}
$$

and $\Delta \eta_{2}$ varies symmetrically about $\phi=0^{\circ}$. Because all the inner equations of system (2) are identical, the same analytical results (3) and (4) are obtained with the following system of three elements:

$$
\begin{aligned}
& \mathrm{Y}_{\mathrm{V}} \Delta \mathrm{V}_{1}+\mathrm{Y}_{\omega} \Delta \omega+\mathrm{Y}_{\eta} \Delta \eta_{1}=-\mathrm{Y}_{\mathrm{e}}-\mathrm{Y}_{\mathrm{nb}} \mathrm{e}^{\mathrm{j} \phi} \equiv \mathrm{Y}_{\text {edge } \_1}(\phi) \\
& \mathrm{Y}_{\mathrm{V}} \Delta \mathrm{V}_{2}+\mathrm{Y}_{\omega} \Delta \omega=-\mathrm{Y}_{\mathrm{nb}} \mathrm{e}^{-\mathrm{j} \phi}-2 \mathrm{Y}_{\mathrm{e}}-\mathrm{Y}_{\mathrm{nb}} \mathrm{e}^{\mathrm{j} \phi} \equiv \mathrm{Y}_{\text {inner }}(\phi) \\
& \mathrm{Y}_{\mathrm{V}} \Delta \mathrm{V}_{3}+\mathrm{Y}_{\omega} \Delta \omega+\mathrm{Y}_{\eta} \Delta \eta_{\mathrm{n} 3}=-\mathrm{Y}_{\mathrm{nb}} \mathrm{e}^{-\mathrm{j} \phi}-\mathrm{Y}_{\mathrm{e}} \equiv \mathrm{Y}_{\text {edge_2 }}(\phi)
\end{aligned}
$$

Furthermore, all the amplitude increments of the inner oscillators are the same and agree with the predictions from (5). Expressions (3) and (4) provide insight into the system behavior. As shown in (3), the oscillation-frequency deviation $\Delta \omega$ varies sinusoidally with $\phi$ about an offset value $\Delta \omega_{0}$, that is: $\Delta \omega(\phi)=\Delta \omega_{0}+\mathrm{W} \cos (\phi)$. The offset $\Delta \omega_{0}$ comes from the change in the oscillator load due to the "self-coupling" element $Y_{\mathrm{e}}$. It increases with $\left|\mathrm{Y}_{\mathrm{e}}\right|$ and decreases with $\left|\mathrm{Y}_{\omega}\right|$, thus, with the oscillator quality factor. The magnitude $|\mathrm{W}|$ is zero for $\sin \left(\alpha_{\mathrm{v}, \mathrm{nb}}\right)=0$ and otherwise increases with $\left|Y_{\mathrm{nb}}\right|$ and decreases with $\left|\mathrm{Y}_{\omega}\right|$. Note that according to [9], a stable free-running oscillation must fulfill $\sin \alpha_{v, \omega}>0$. On the other hand, for maximum parameter excursion at $\pm 90^{\circ}$, one should have $\sin \left(\alpha_{\mathrm{v}, \mathrm{nb}}\right)=0$, which provides a parameter deviation equal to the constant offset in (4) at $\phi=0^{\circ}$, and increments of $\pm\left|Y_{n b}\right| /\left|Y_{\eta}\right| \sin \alpha_{v, \eta}$ at $\pm 90^{\circ}$.

The method has been applied to the system in Fig. 1. Initially, the system coupling strength has been evaluated, obtaining the locking bandwidth of two oscillator elements: $\Delta \omega_{\text {lock }}=2\left|\mathrm{Y}_{\mathrm{nb}}\right| /\left(\left|\mathrm{Y}_{\omega}\right| \sin \alpha_{\mathrm{v}, \omega}\right)$. With $\mathrm{R}_{\mathrm{c}}=330 \Omega$ and $l=33.9 \mathrm{~mm}$ (one wavelength), this provides $\Delta \mathrm{f}_{\text {lock }}=29 \mathrm{MHz}$, whereas full $\mathrm{HB}$ at circuit level provides $\Delta \mathrm{f}_{\text {lock,HB}}=26 \mathrm{MHz}$. In Fig. 2, results from (3) to (5) are compared with those obtained with $\mathrm{HB}$ for $n=3,4,5,6$. HB convergence was not possible with $n>6$.

For the stability analysis, small increments in all the oscillator amplitudes and phase values, as well as the oscillation frequency, are considered. System (5) is linearized about the solution with constant phase shift $\phi$, which provides the following linear-time-invariant (LTI) system:

$$
\Delta \dot{\bar{X}}(\mathrm{t})=-\left[\begin{array}{cccccc}
\frac{Y_{\omega}^{\mathrm{i}}}{\mathrm{V}_{0}} & 0 & 0 & \mathrm{Y}_{\omega}^{\mathrm{r}} & 0 & 0 \\
-\frac{Y_{\omega}^{\mathrm{r}}}{\mathrm{V}_{0}} & 0 & 0 & \mathrm{Y}_{\omega}^{\mathrm{i}} & 0 & 0 \\
0 & \frac{Y_{\omega}^{\mathrm{i}}}{\mathrm{V}_{0}} & 0 & 0 & \mathrm{Y}_{\omega}^{\mathrm{r}} & 0 \\
0 & -\frac{\mathrm{Y}_{\omega}^{\mathrm{r}}}{\mathrm{V}_{0}} & 0 & 0 & \mathrm{Y}_{\omega}^{\mathrm{i}} & 0 \\
0 & 0 & \frac{\mathrm{Y}_{\omega}^{\mathrm{i}}}{\mathrm{V}_{0}} & 0 & 0 & \mathrm{Y}_{\omega}^{\mathrm{r}} \\
0 & 0 & -\frac{\mathrm{Y}_{\omega}^{\mathrm{r}}}{\mathrm{V}_{0}} & 0 & 0 & \mathrm{Y}_{\omega}^{\mathrm{i}}
\end{array}\right]\left[\begin{array}{cccccc}
\mathrm{Y}_{\mathrm{v}}^{\mathrm{r}} & 0 & 0 & \mathrm{a} & -\mathrm{a} & 0 \\
\mathrm{Y}_{\mathrm{v}}^{\mathrm{i}} & 0 & 0 & \mathrm{~b} & -\mathrm{b} & 0 \\
0 & \mathrm{Y}_{\mathrm{v}}^{\mathrm{r}} & 0 & \mathrm{c} & \mathrm{d} & -\mathrm{a} \\
0 & \mathrm{Y}_{\mathrm{v}}^{\mathrm{i}} & 0 & \mathrm{e} & \mathrm{f} & -\mathrm{b} \\
0 & 0 & \mathrm{Y}_{\mathrm{v}}^{\mathrm{r}} & 0 & \mathrm{c} & -\mathrm{c} \\
0 & 0 & \mathrm{Y}_{\mathrm{v}}^{\mathrm{i}} & 0 & \mathrm{e} & -\mathrm{e}
\end{array}\right] \Delta \overline{\mathrm{X}}(\mathrm{t})
$$


where

$$
\begin{aligned}
& \mathrm{a}=\mathrm{Y}_{\mathrm{nb}}^{\mathrm{i}} \cos (\phi)+\mathrm{Y}_{\mathrm{nb}}^{\mathrm{r}} \sin (\phi) ; \mathrm{b}=-\mathrm{Y}_{\mathrm{nb}}^{\mathrm{r}} \cos (\phi)+\mathrm{Y}_{\mathrm{nb}}^{\mathrm{i}} \sin (\phi) \\
& \mathrm{c}=-\mathrm{Y}_{\mathrm{nb}}^{\mathrm{i}} \cos (\phi)+\mathrm{Y}_{\mathrm{nb}}^{\mathrm{r}} \sin (\phi) ; \mathrm{e}=\mathrm{Y}_{\mathrm{nb}}^{\mathrm{r}} \cos (\phi)+\mathrm{Y}_{\mathrm{nb}}^{\mathrm{i}} \sin (\phi) \\
& \mathrm{d}=2 \mathrm{Y}_{\mathrm{nb}}^{\mathrm{i}} \cos (\phi) ; \mathrm{f}=-2 \mathrm{Y}_{\mathrm{nb}}^{\mathrm{r}} \cos (\phi)
\end{aligned}
$$

and the vector $\Delta \overline{\mathrm{X}}(\mathrm{t})$ is defined as $\Delta \overline{\mathrm{X}}=\left(\Delta \overline{\mathrm{V}}^{\mathrm{t}} \Delta \bar{\phi}^{\mathrm{t}}\right)$. Due to the system autonomy, one of the eigenvalues is $\lambda_{1}=0$. Some algebraic manipulation provides the following approximate expression for the real part of the dominant eigenvalue $\lambda_{2}$ :

$$
\lambda_{2}^{\mathrm{r}} \cong \frac{3\left|\mathrm{Y}_{\mathrm{nb}}\right| \cos \alpha_{\mathrm{v}, \mathrm{nb}} \sin \alpha_{\mathrm{v}, \omega}}{2\left|\mathrm{Y}_{\omega}\right|}=\frac{3}{4} \Delta \omega_{\mathrm{lock}} \cos \alpha_{\mathrm{v}, \mathrm{nb}}\left(\sin \alpha_{\mathrm{v}, \omega}\right)^{2}(8)
$$

where $\lambda_{2}^{r}$ has been related to the locking bandwidth. The dominant eigenvalues of (6) can only have a zero real part at $\phi= \pm 90^{\circ}$, where the $\cos ()$ functions are zero. Then, with $\cos \alpha_{\mathrm{v}, \mathrm{nb}}<0 \quad\left(\cos \alpha_{\mathrm{v}, \mathrm{nb}}>0\right)$, the stable interval is $-90^{\circ}<\phi<90^{\circ}\left(90^{\circ}<\phi<270^{\circ}\right)$. One possible design goal is to minimize the oscillation-frequency variation $(\Delta \omega)$ when changing $\phi$ through the stable phase shift interval $-90^{\circ}$ to 90 . This is obtained by imposing the two conditions: $\sin \alpha_{\mathrm{v}, \mathrm{nb}}=0$ and $\cos \alpha_{\mathrm{v}, \mathrm{nb}}<0$. These conditions are fulfilled for the coupling line length $l_{\text {opt }}=37.5 \mathrm{~mm}$, different from the common choice of one wavelength.
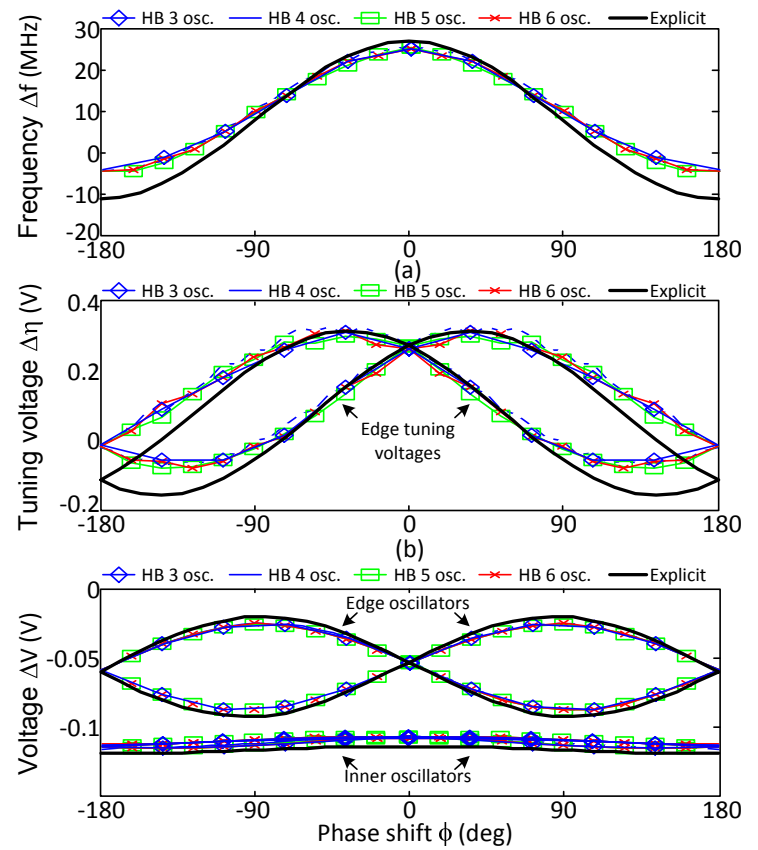

(c)

Fig. 2 Comparison between results obtained with the two-level analysis (dashed line) and with full HB circuit-level simulations. Various numbers of oscillator elements $(n=3,4,5,6)$ have been considered. (a) Frequency deviation. (b) Tuning voltage. (c) Amplitude distribution.
The explicit expressions (3)-(4) can be easily introduced in commercial HB simulators to evaluate the impact of any modification of the oscillator design at circuit level, on the coupled-system operation. In this way, only the individual oscillator is simulated, instead of the coupled system. For illustration, variations in the output inductance $\mathrm{L}_{d}$ (Fig. 1) are considered in Fig. 3, which is swept down from its original value. At each step, three actions are carried out. (a) Simulation at circuit level of the new free-running oscillatory solution. To keep the desired oscillation frequency $\mathrm{f}_{0}$, the $A G$ frequency is fixed as $\mathrm{f}_{\mathrm{AG}}=\mathrm{f}_{0}$ and the output capacitance $\mathrm{C}_{\mathrm{d}}$, the gate inductor $\mathrm{L}_{\mathrm{g}}$ and $\mathrm{A}_{\mathrm{AG}}$ are optimized to fulfill the non-perturbation condition $\mathrm{Y}_{\mathrm{AG}}=0$. (b) After convergence, a new set of derivatives $Y_{V}, Y_{\omega}, Y_{\eta}$ is calculated through finite differences. (c) These derivatives are introduced in the outer-level formulation to optimize the coupled-system performance. Fig. 3(a) shows the variation of $\angle Y_{v}$ and $\sin \alpha_{v, \omega}$. In the outerlevel analysis, the coupling-line length is swept, selecting the value that fulfills the double condition $\sin \alpha_{\mathrm{v}, \mathrm{nb}}=0$ and $\cos \alpha_{v, n b}<0$. See an example in Fig. 3(b), for a particular $L_{d}$ value. In Fig. 4 results obtained with the original coupling-line length $(l=33.9 \mathrm{~mm})$ and with the optimized value $\left(l_{\mathrm{opt}}=37.5 \mathrm{~mm}\right)$ are compared. In the two cases, the system was analyzed with full $\mathrm{HB}$, at circuit level. The optimized design enables minimum frequency deviation and maximum parameter excursions at $\pm 90^{\circ}$. Measurements of frequency derivation and tuning voltages have been superimposed.
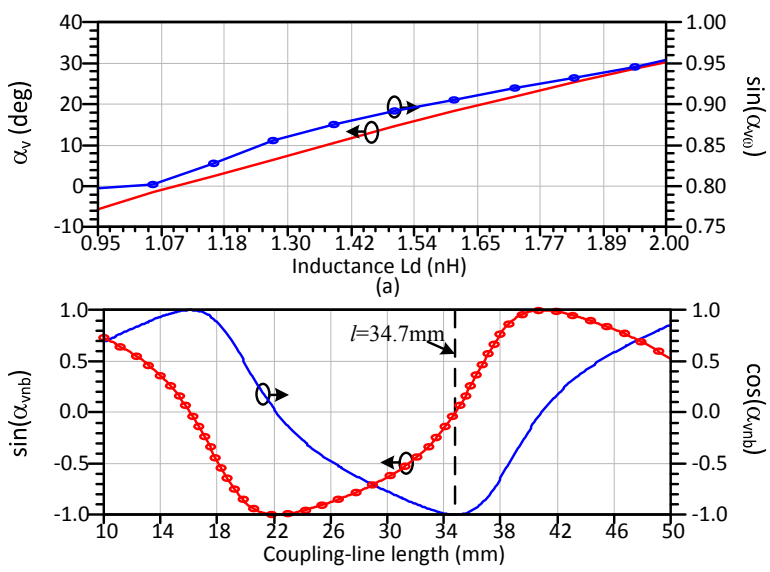

(b)

Fig. 3 Two-level analysis of the coupled-oscillator system in Fig. 1. (a) Variation of the angle of $Y_{v}$ and function $\sin \alpha_{v, \omega}$ versus the inductance $L_{d}$. (b) Example of graphical determination of the optimum coupling-line length.

\section{EXTENSION TO INJECTION-LOCKED OPERATION}

In injection-locked conditions, an external signal is introduced at any of the coupled oscillators and the new system is:

$$
\begin{aligned}
& \mathrm{M}_{\mathrm{X}} \overline{\mathrm{X}}=\overline{\mathrm{Y}}_{\mathrm{c}}(\phi)+\overline{\mathrm{Y}}_{\mathrm{s}}\left(\mathrm{I}_{\mathrm{s}}, \phi_{\mathrm{s}}-\phi\right), \\
& \overline{\mathrm{Y}}_{\mathrm{s}}\left(\mathrm{I}_{\mathrm{s}}, \phi \phi_{\mathrm{s}}, \phi\right)=\left(0 \cdots \frac{\mathrm{I}_{\mathrm{s}}}{\mathrm{V}_{0}} \mathrm{e}^{\mathrm{j}\left(\phi_{\mathrm{s}}-(\mathrm{k}-1) \phi\right)} \cdots 0\right)^{\mathrm{t}}
\end{aligned}
$$

where $I_{s}, \omega_{\mathrm{s}}$ and $\phi_{\mathrm{s}}$ are, respectively, the amplitude, frequency and phase of the injection source. It is immediately derived 
from (9) that the locking bandwidth is zero when the injection source is connected to any of the two outermost oscillators (tuned to change $\phi$ ). When connected to any of the other elements, the bandwidth is independent of the choice of the injected oscillator $(\mathrm{k})$. The band is the same for 3 oscillator elements and for an arbitrary number $\mathrm{n}$ and corresponds to:

$$
\begin{aligned}
& \Delta \omega_{\mathrm{s}, 1}=\Delta \omega_{0}+\frac{2\left|\mathrm{Y}_{\mathrm{nb}}\right|\left|\sin \alpha_{\mathrm{v}, \mathrm{nb}}\right|}{\left|\mathrm{Y}_{\omega}\right| \sin \alpha_{\mathrm{v}, \omega}}-\frac{\mathrm{I}_{\mathrm{s}}}{\mathrm{V}_{\mathrm{o}}\left|\mathrm{Y}_{\omega}\right| \sin \alpha_{\mathrm{v}, \omega}} \\
& \Delta \omega_{\mathrm{s}, 2}=\Delta \omega_{0}-\frac{2\left|\mathrm{Y}_{\mathrm{nb}}\right|\left|\sin \alpha_{\mathrm{v}, \mathrm{nb}}\right|}{\left|\mathrm{Y}_{\omega}\right| \sin \alpha_{\mathrm{v}, \omega}}+\frac{\mathrm{I}_{\mathrm{s}}}{\mathrm{V}_{\mathrm{o}}\left|\mathrm{Y}_{\omega}\right| \sin \alpha_{\mathrm{v}, \omega}}
\end{aligned}
$$

where $\Delta \omega_{\text {free }}(\phi)$ refers to the frequency deviation in freerunning conditions, given in (3). Within the locking band, the phase shift $\phi$ between adjacent oscillator elements can vary in the full interval $-180^{\circ} \leq \phi \leq 180^{\circ}$ (although not all these values will correspond to stable solutions). Expression (10)(b) evidences the opposed effects of oscillator coupling and injection locking. Maximum bandwidth is obtained for $\sin \alpha_{\mathrm{v}, \mathrm{nb}}=0$, which minimizes the oscillation-frequency deviation due to coupling effects [see (3)]. At each constant $\Delta \omega_{\mathrm{s}}$, the phase values $\phi_{\mathrm{s}}$ and $\phi$ fulfill the relationship:

$$
\Delta \omega_{\mathrm{s}}=\Delta \omega_{\text {free }}(\phi)+\frac{\mathrm{I}_{\mathrm{s}} \sin \left(\phi_{\mathrm{s}}-(\mathrm{k}-1) \phi-\alpha_{\mathrm{v}}\right)}{\mathrm{V}_{\mathrm{o}}\left|\mathrm{Y}_{\omega}\right| \sin \alpha_{\mathrm{v}, \omega}}
$$

Linearizing the injection-locked coupled-system equations, one obtains that the stable phase-shift interval is maximized for $\sin \alpha_{\mathrm{v}, \mathrm{nb}}=0$. If $\cos \alpha_{\mathrm{v}, \mathrm{nb}}<0$, this interval corresponds to $-90^{\circ}<\phi<90^{\circ}$. In Fig. 5, the inter-stage phase shift $\phi=45^{\circ}$ is maintained through the whole synchronization bandwidth. Results are successfully compared with the numerical technique in [5] and with costly HB simulations.

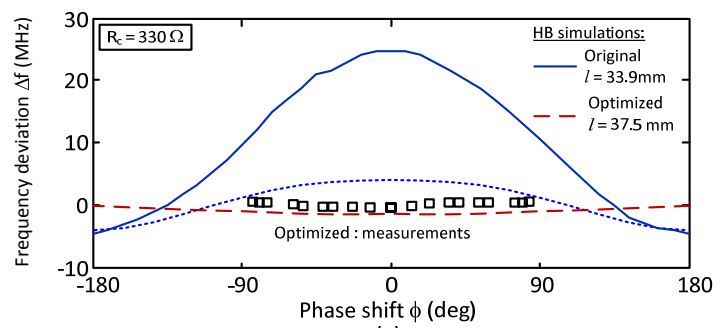

(a)

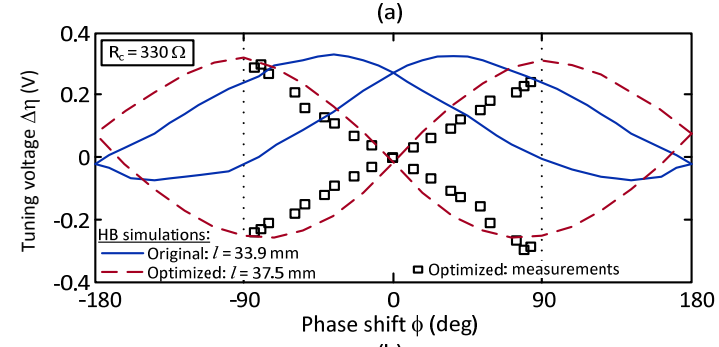

(b)

Fig. 4 Comparison of the original design $(l=33.9 \mathrm{~mm})$ and an optimum design fulfilling $\sin \alpha_{\mathrm{v}, \mathrm{nb}}=0$ and $\cos \alpha_{\mathrm{v}, \mathrm{nb}}<0 \quad(l=37.5 \mathrm{~mm})$. (a) Frequency deviation. (b) Tuning parameters.

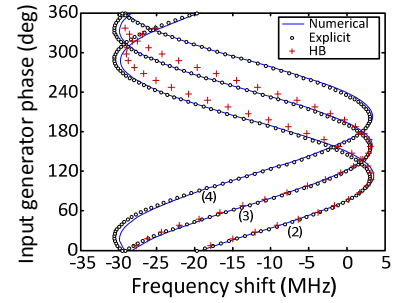

(a)

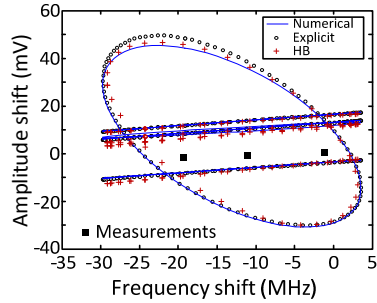

(b)
Fig. 5. Two-level analysis with an injection signal in a design fulfilling $\sin \alpha_{\mathrm{v}, \mathrm{nb}}=0$ and $\cos \alpha_{\mathrm{v}, \mathrm{nb}}<0$. Inter-stage phase shift is fixed at $\phi=45^{\circ}$. (a) Phase shift $\phi_{s}$ versus $\omega_{\mathrm{S}}$ for $\mathrm{n}=5$ when introducing the injection signal at different oscillator elements. (b) Amplitude distribution for $\mathrm{n}=7$.

\section{CONCLUSIONS}

A two-level simulation methodology for coupled-oscillator systems has been presented. The fact that a system of 3 elements constitutes a valid model for a system with an arbitrary number $\mathrm{n}$ of elements has been demonstrated. Mathematical conditions for minimum frequency deviation and unambiguous tuning-parameter variation have been derived. The analysis methodology has been extended to the case of coupled-oscillator systems with injection signals.

\section{ACKNOWLEDGMENT}

This work was supported by the Spanish Ministry of Economy and Competitiveness under Contract TEC2011-29264-C03-01.

\section{REFERENCES}

[1] R. A. York, "Nonlinear analysis of phase relationships in quasi-optical oscillator arrays", IEEE Trans. Microw. Theory Techn., vol. 41, no. 10, 1993 pp: $1799-1809$

[2] P. Liao, R. A. York, "A new phase-shifterless beam-scanning technique using arrays of coupled oscillators", IEEE Trans. Microw. Theory Techn., vol. 41, no. 10, pp. 1810-1815, Oct. 1993.

[3] T. Heath, "Beam steering of nonlinear oscillator arrays through manipulation of coupling phases," IEEE Trans. on Antennas and Propagation, vol. 52, no. 7, pp. 1833-1842, Jul. 2004.

[4] A. Suárez, A. Collado, F. Ramírez, "Harmonic-balance techniques for the design of coupled-oscillator systems in both unforced and injectionlocked operation”, 2005 IEEE MTT-S, Long Beach, Jun., 2005.

[5] A. Georgiadis, A. Collado, A. Suarez, "New Techniques for the Analysis and Design of Coupled-Oscillator Systems", IEEE Trans. Microw. Theory Techn., vol. 54, no. 11, Nov. 2006.

[6] A. Suárez, F. Ramírez, S. Sancho, "Stability and Noise Analysis of Coupled-Oscillator Systems,” IEEE T-MTT, vol. 59, no. 4, pp. 10321046, 2011.

[7] F. Ramírez, E. de Cos, A. Suárez, "Nonlinear analysis tools for the optimized design of harmonic-injection frequency dividers", IEEE Trans. Microw. Theory Techn., vol. 51, no. 6, Jun. 2003, pp. 1752-1762.

[8] A. Suárez, S. Sancho, F. Ramírez, General formulation for the analysis of injection-locked coupled-oscillator systems, IEEE T-MTT, vol. 61, no. 12,2013

[9] K. Kurokawa, "Some basic characteristics of broadband negative resistance oscillators," The Bell System Technical Journal, vol. 48, pp. 1937-1955, July-August. 1969. 